\title{
Recurrent Intrahepatic Cholestasis
}

National Cancer Institute

\section{Source}

National Cancer Institute. Recurrent Intrahepatic Cholestasis. NCI Thesaurus. Code C84401.

Reappearance of cholestasis caused by obstruction within the liver. 